Gut, 1976, 17, 41-47

\title{
Review of five cases of early gastric carcinoma
}

\author{
D. I. FEVRE, P. H. R. GREEN, P. J. BARRATT, AND G. S. NAGY \\ From the Royal North Shore Hospital of Sydney, St Leonards, N.S.W., Australia
}

SUMMARY Five cases of early gastric carcinoma (EGC) were seen in 12 months. The clinical features did not provide guide-lines to diagnosis, which depends on air contrast barium studies, endoscopic recognition of early malignancy, adequate biopsies, and their interpretation. Malignancy was present in an average of $60 \%$ of the biopsies taken. The most common type of lesion was IIc. The superficial nature of the malignancy was predicted at endoscopy in four cases but was unsuspected in one case which was considered to be advanced carcinoma until the resected specimen was thoroughly examined microscopically. The excellent prognosis of EGC was discussed. Only conservative surgery need be performed if the superficial nature of the lesion is recognized before operation.

Early gastric cancer (EGC) can be defined as carcinoma confined to mucosa or submucosa without infiltration into the muscularis propria (Murakami, 1971). This condition is well recognized in Japan where mass screening is used to detect carcinoma because of its high prevalence (Segi, 1969), but is seldom recognized in Australia, the United Kingdom, and U.S.A., judging by the sparsity of references in the literature. The high five year survival rate of surgically treated EGC (more than $95 \%$ ) compared with that of advanced gastric cancer (approximately 30\%) (Ransom, 1953; Muto, 1962) provides the stimulus for making a diagnosis at this stage of disease.

The detection of this early lesion requires team work between expert radiologists giving good air contrast barium studies, experienced endoscopists aware of the condition, and histopathologists capable of interpreting the small biopsy specimens.

This paper describes our experience of early gastric carcinoma during a 12 month period. During this period, five cases of early gastric cancer were seen among a total of 26 cases of gastric carcinoma on whom endoscopy was performed.

\section{Methods}

RADIOLOGY

Air contrast barium studies were performed in all our patients as outlined by Shirakabe (1971).

ENDOSCOPY

Olympus gastroscope type GFB was used on all

Received for publication 2 October 1975 patients except one: Olympus JFB duodenoscope was used in case 5. All patients had topical lignocaine $4 \%$ applied to the pharynx together with 5 to $10 \mathrm{mg}$ intravenous diazepam as premedication.

The classification of lesions considered to be early gastric cancer was that used by the Japan Society for Gastroenterological Endoscopy, and is shown in Fig. 1.

At least seven biopsies were taken from all lesions with emphasis on edges of the depressed areas.

\section{PATHOLOGY}

Preparation and examination of biopsy material Biopsies were placed on paper and each biopsy put

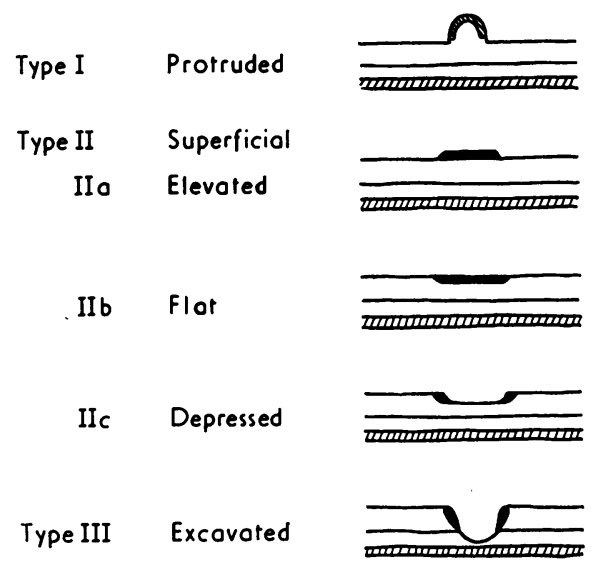

Fig. 1 Endoscopic classification of early gastric carcinoma. 


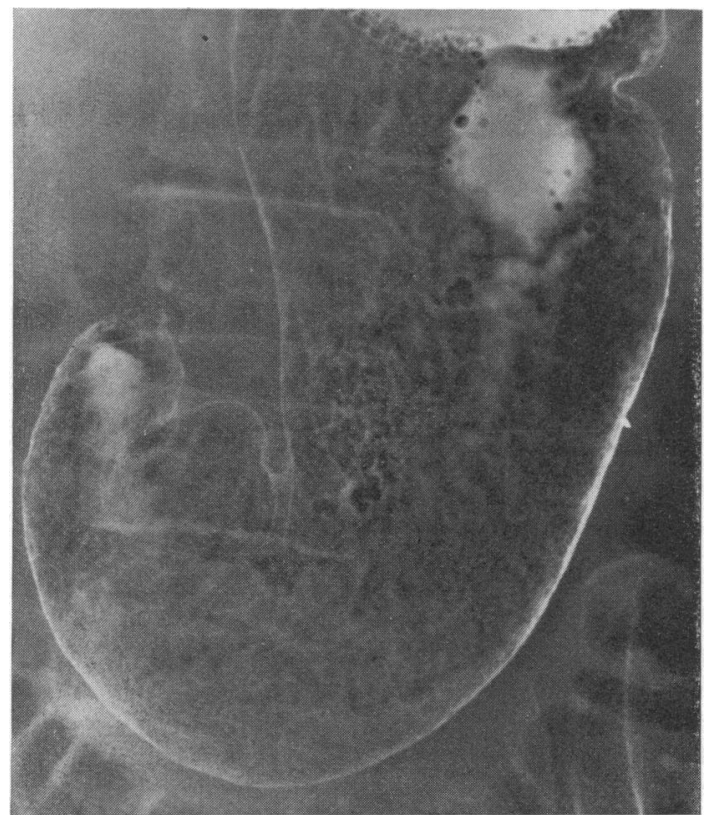

Fig 2

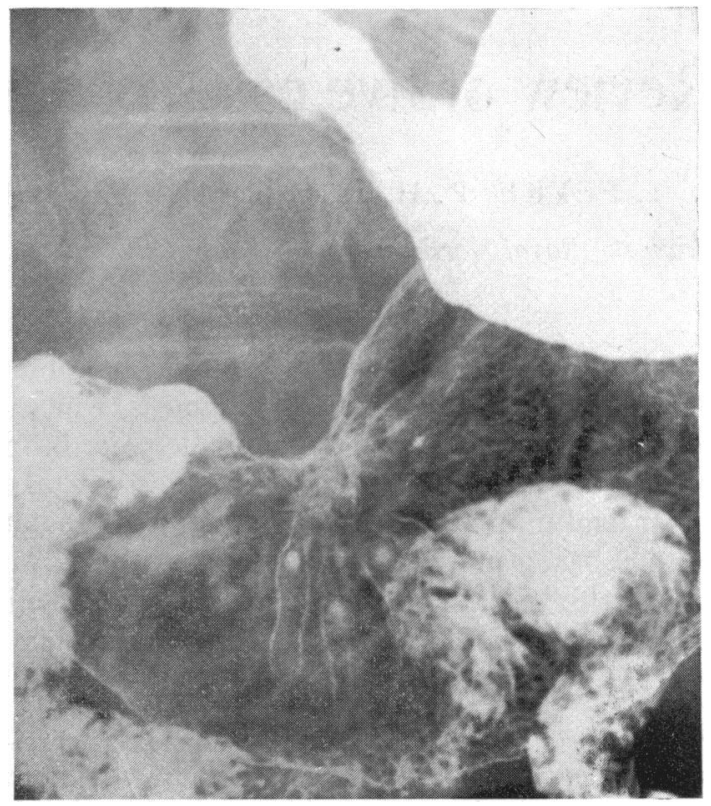

Fig 3

Fig. 2 Case 2. Radiograph showing well-circumscribed area of elevated mucosa adjacent to the lesser curvature.

Fig. 3 Case 4. Radiograph showing superficial ulceration and induration. There is mucosal deformity with thickening of the termination of the folds, most of which fall short of the area of induration and ulceration.

into $10 \%$ formol saline in separate numbered containers. Histological sections stained with haematoxylin and eosin were examined.

In doubtful instances of poorly differentiated carcinoma a PAS/Alcian Blue stain was used to show individual malignant cells in the lamina propria.

\section{Preparation of resected stomach}

The resected stomachs were brought to the laboratory immediately on removal and opened along the greater curvature. They were then laid out flat, pinned, and photographed. They were fixed for at least 48 hours.

Blocks were then cut through the entire areas suspected of being involved by carcinoma. The specimen was photographed with blocks in place and each block was carefully numbered. Lymph nodes were also sectioned. Sections were then cut and stained routinely.

CLASSIFICATION

\section{Macroscopic}

The gross appearances were described according to the classification of the Japan Society for Gastroenterological Endoscopy (Fig. 1).

\section{Microscopic}

All lesions were adenocarcinoma and were described according to the degree of differentiation. They were

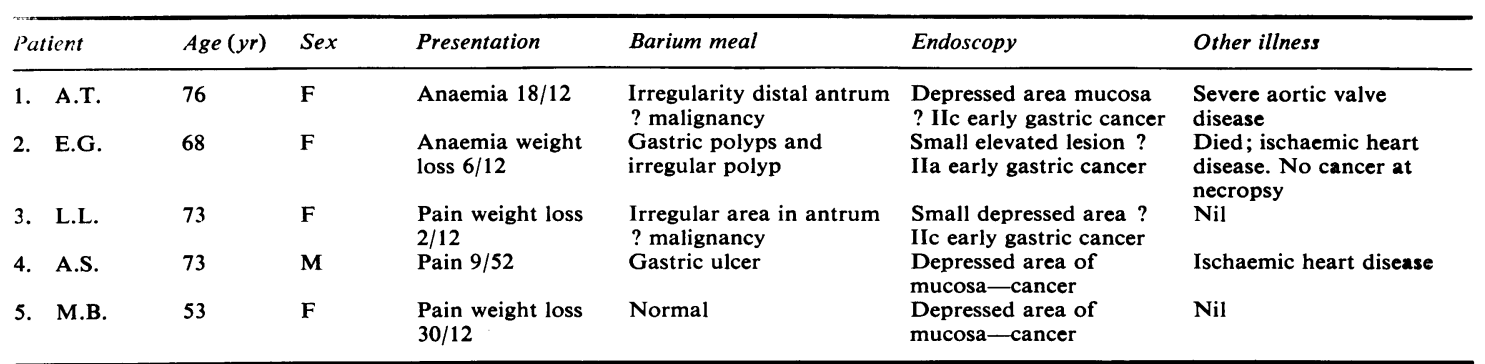

Table 1 Presenting symptoms and results of investigations 


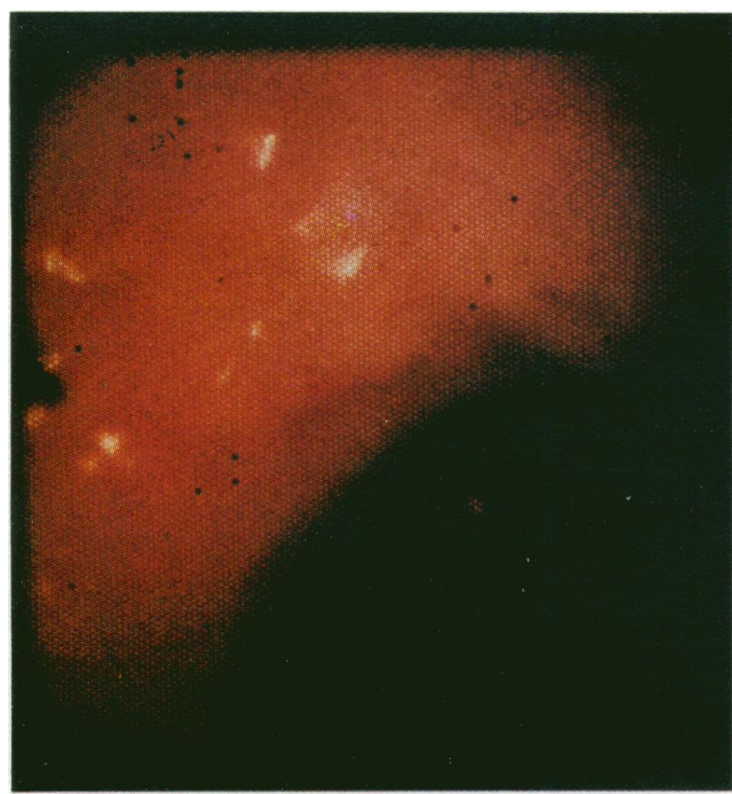

Fig. 4 Case 2. Endoscopy showing a non-ulcerated elevated mucosal lesion (type IIa).

predominantly either well differentiated or poorly differentiated. The well-differentiated tumours had either a tubular or a papillary pattern. The poorly differentiated tumours showed malignant cells spreading singly in the lamina propria. A variable number of these cells were mucus secreting and sometimes had a classical signet ring appearance.

\section{Results}

The presenting symptoms and the results of investigations on each patient are summarized in Table 1.

\section{RADIOLOGY}

The possibility of malignancy on barium studies was suggested in two of the five cases ( 1 and 3 ), while endoscopy was suggested for clarification by the

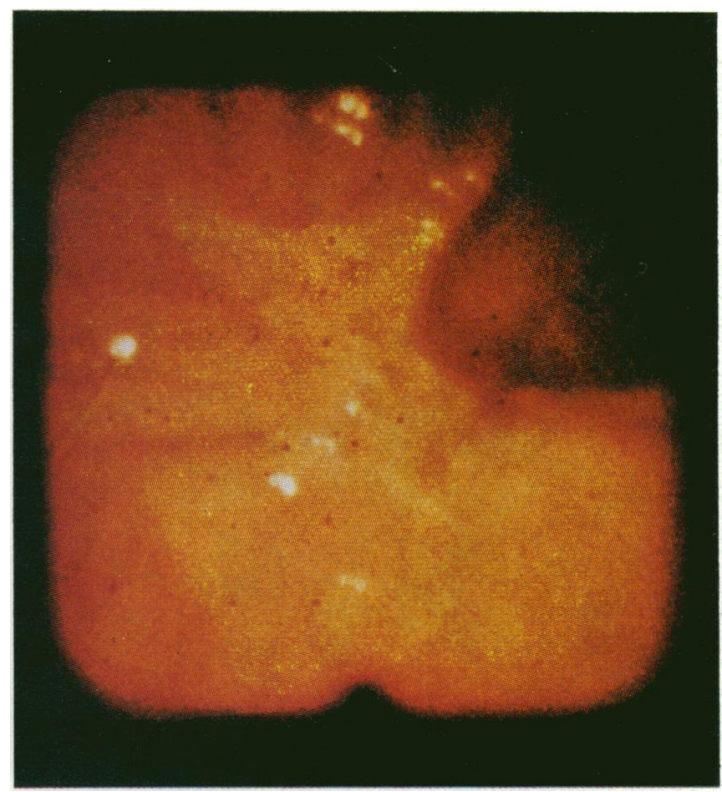

Fig. 5 Case 4. Endoscopy showing a depressed area of mucosa (type IIc lesion).

radiologist in cases 1,2 , and 3 . Case 2 showed an elevated area of gastric mucosa (Fig. 2). Case 4 was reported initially as a gastric ulcer which, on retrospective examination of the films, had the features of a malignant ulcer. A repeat barium meal after endoscopy showed many features of malignancy (Fig. 3). Case 5 had two barium meals reported as normal during the period of her illness; review of these films, however, did not show an adequate mucosal pattern of the involved region.

\section{ENDOSCOPY}

At endoscopy, all the patients were considered to have malignant lesions. Cases 1,2 , and 3 were considered to be early gastric cancer, while cases 4 and 5 were considered to be malignant, early gastric carcinoma being reported only after examination of

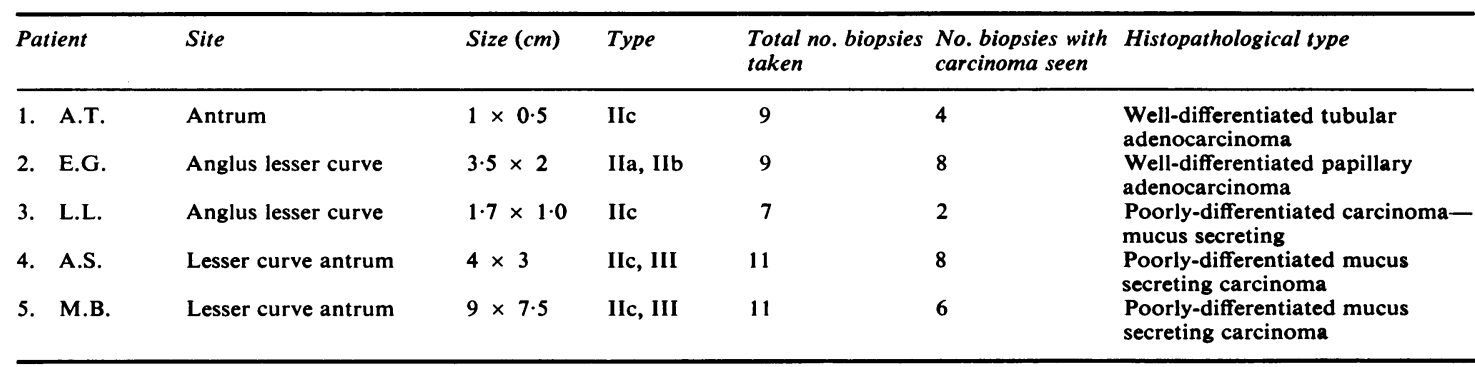

Table 2 Relation between number of biopsies and carcinoma 


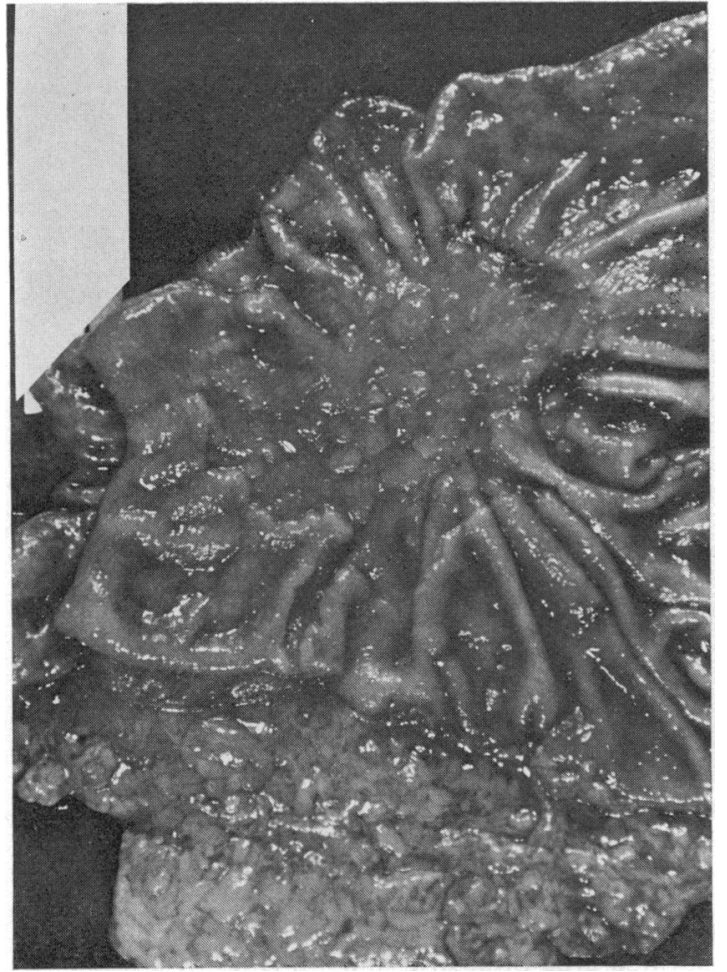

Fig. 6 Case 5. Resected stomach showing large IIC early gastric cancer. the resected specimen. Figure 4 shows the elevated appearance (IIa type lesion) seen at endoscopy in case 2 . Figure 5 shows the depressed llc type lesion of case 4 .

\section{PATHOLOGY}

\section{Biopsy material}

Table 2 shows the relation between the number of biopsies taken and those showing carcinoma.

\section{RESECTED STOMACHS}

\section{Macroscopic}

Four of the five lesions were either totally or predominantly IIc in type. The others were IIa and Ilb (Table 2). This corresponded well with opinion on endoscopy.

Figure 6 shows the unfixed stomach from case 5 in which the IIc lesion measured $9 \times 7.5 \mathrm{~cm}$.

\section{Microscopic}

Two carcinomas were well differentiated and three were poorly differentiated. They were all confined to the mucosa. There was no evidence of lymphatic involvement.

In the two largest lesions, cases 4 and 5 , most sections showed carcinoma toward the periphery of the depressed area (Fig. 7) but towards the centre there were areas of mucosa in which carcinoma was not visible. In some places the mucosa was eroded (Fig. 8) in others it was thin but intact (Fig. 9).

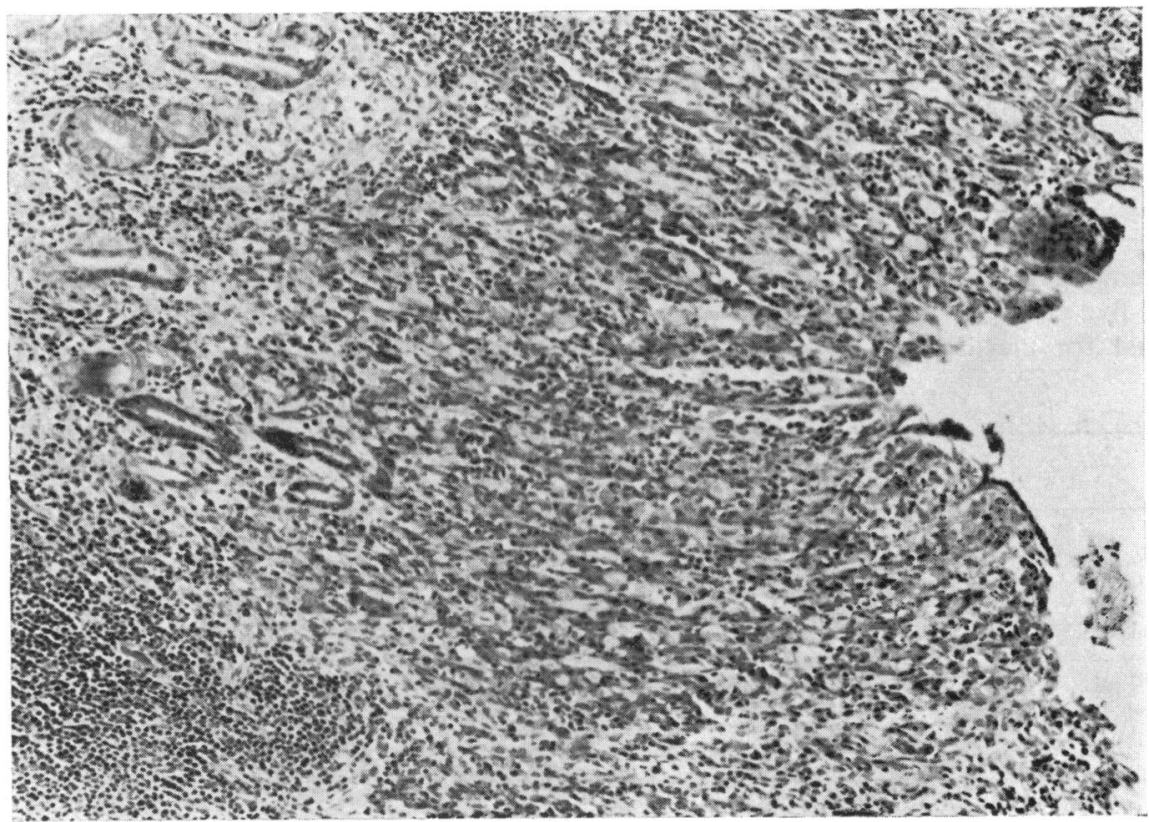

Fig. 7 Case 4. Edge of depressed area. Poorly differentiated carcinoma is present in the upper part of the mucosa. $H$ and $E$ $\times 300$. 

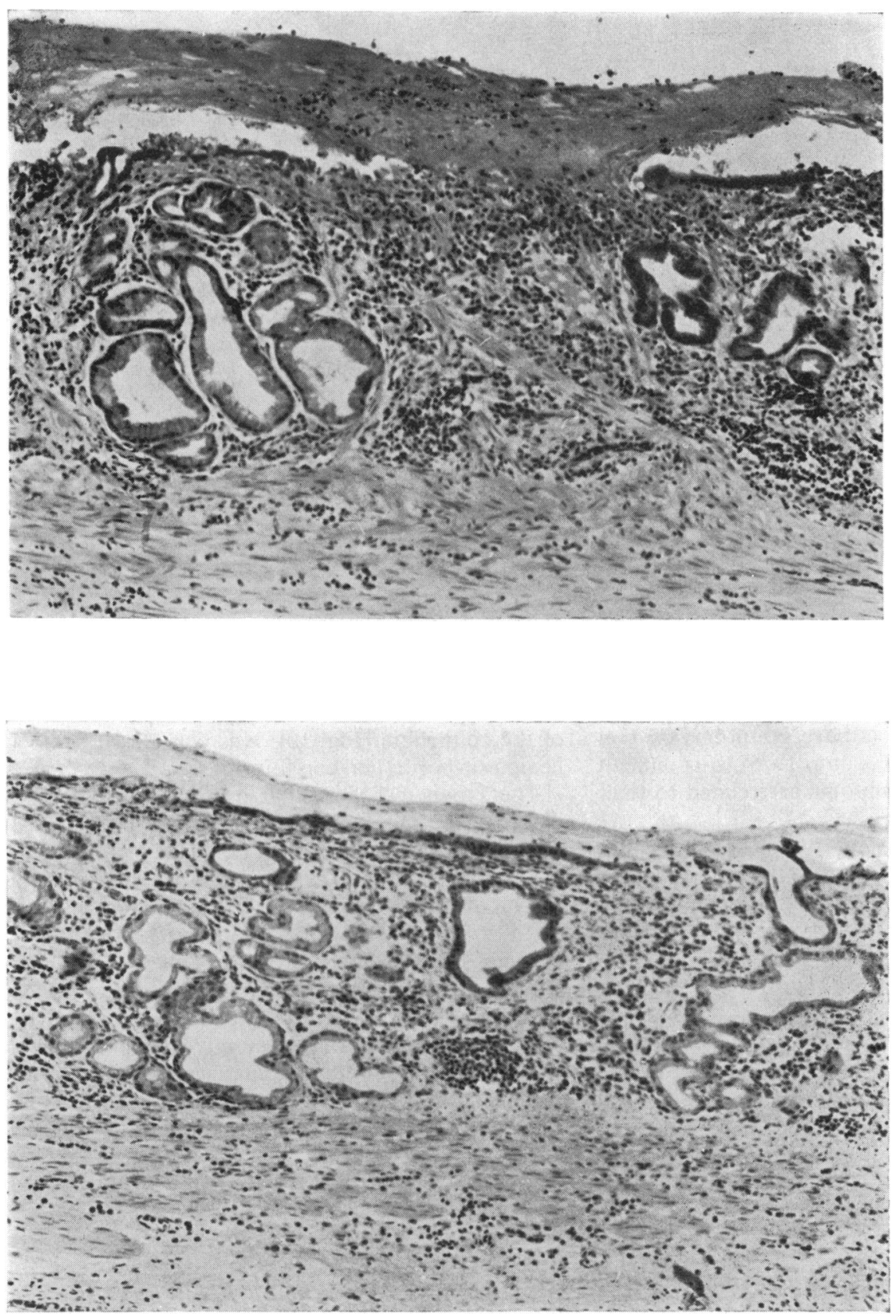

Fig. 8 Case 8. Thin eroded mucosa from the central part of the depressed area. No carcinoma is present. $H$ and $E$ $\times 300$.
Fig. 9 Case 8. Thin intact mucosa from the central part of the depressed area. No carcinoma is present. Biopsies taken from areas shown in Fig. 8 and Fig. 9 would have been misleading. $H$ and $E \times 300$.

\section{Discussion}

\section{CLINICAL FEATURES}

Three of the patients presented with abdominal pain, the nature being consistent with peptic ulceration.
The other two patients had anaemia which initiated investigation of their gastrointestinal tract. In contrast, most Japanese patients are asymptomatic and are diagnosed on mass screening techniques using air contrast barium studies or gastro-camera studies. 


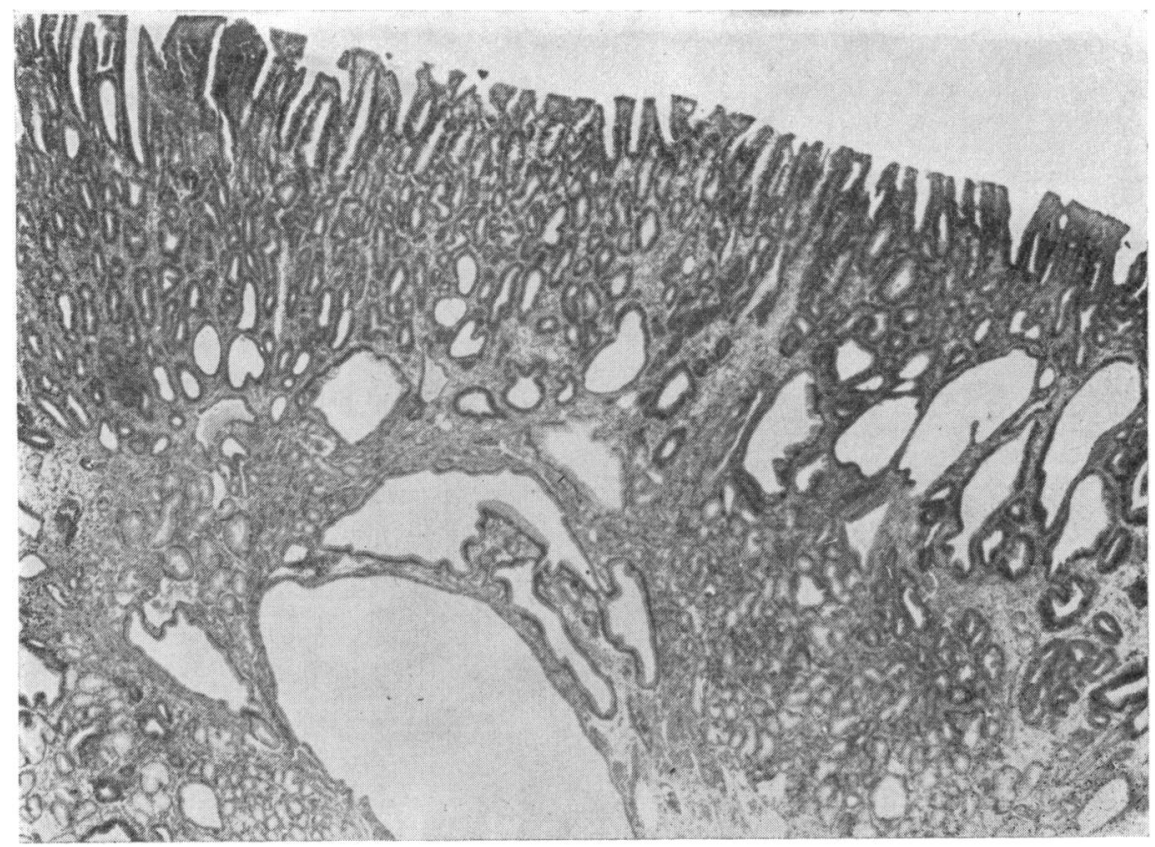

Fig. 10 A polypoid borderline lesion which the Japanese call ATP (an atypical epithelium of the stomach). The glands are crowded and cellular atypia is present but not as marked as in adenocarcinoma. $H$ and $E \times 75$.
Only $40 \%$ of their patients have symptoms on presentation, usually pain (Kasumi, 1975). It is difficult to know whether the symptoms are related to their gastric lesions.

The long duration of symptoms in case 5 is not incompatible with EGC, because the lesion may be present for five to 10 years before invasion beyond the mucosa occurs (Sano, 1972).

\section{PATHOLOGY}

Table 2 shows that carcinoma was present in only about half the biopsies taken. This is partly due to dfficulty in taking biopsies but mainly to the fact that part of the depressed area is devoid of carcinoma. Histological examination clearly demonstrated that the most likely place to find carcinoma in a IIc type lesion was close to the edge of the depressed area.

As far as interpretation of biopsy material is concerned, carcinoma can be reported with confidence if individual malignant cells can be seen in the lamina propria. This is found in poorly differentiated carcinoma and was seen in three of these patients.

If the carcinoma is well differentiated, biopsy interpretation is much more difficult. The differential diagnosis includes actively regenerating epithelium and the lesion which Japanese pathologists call ATP, which stands for an atypical epithelium (Sugano et al., 1971; Yokoyama et al., 1974), ATP is a polypoid lesion with atypical features of the glandular pattern of the epithelium (Fig. 10). It is considered to be a benign or borderline condition.

The Japanese believe that it corresponds to dysplasia of the uterine cervix and should be followed up by endoscopy rather than resected.

The main distinguishing features in differentiating carcinoma from degenerating epithelium or ATP are as follows. Regenerating gastric epithelium is usually not sharply demarcated from the surrounding epithelium but shows a more gradual transition than carcinoma does.

In ATP the cellular atypia is not as great as it is in cancer. Also, well-differentiated cancer usually involves the whole layer of the mucosa, whereas ATP may have normal glands below and normal surface epithelium above.

When examining the resected stomach, it is preferable to take blocks from the whole specimen but obviously in many routine laboratories this is not feasible. Alternatively, the entire area involved by carcinoma must be examined until the pathologist is confident that the limits of the lesion have been reached. Otherwise, it is possible that an area of submucosa or intramucosal invasion will be missed.

At surgery, there was no evidence of metastatic carcinoma in our five cases and no evidence was found at necropsy in case 2 .

PROGNOSIS

The prognosis of EGC, as stated earlier, is excellent, 
but, as the disease tends to occur in older patients, other illnesses may be present and death due to these tends to occur in the follow-up period (Kidokoro, 1971).

The recurrence rate of EGC appears to be about $5 \%$ (Hayashida 4.7\%, 1969; Sano 4.5\%, 1971). Important factors affecting the recurrence in EGC are the depth of cancer invasion, lymph node metastases, and vascular invasion. Cancer confined to the mucosa has a very low recurrence rate, submucosal cases show occasional recurrence, and it is in these that metastases to lymph nodes tend to occur (Yamada et al., 1974; Yamada, 1975).

All our patients had extensive gastrectomies short of total gastrectomy. However, there is evidence that more conservative surgery is sufficient (Yamada, 1975).

It can be seen that the prognosis varies inversely with the depth of invasion-that is, the earlier the diagnosis, the better the prognosis. It is probable that early diagnosis can only be made with early endoscopy and we suggest gastrocopy in all patients with abnormalities on barium studies at our hospital. We also endoscope those with normal barium studies who have persistent upper gastrointestinal symptoms.

We are grateful to Dr John Hunt, Director of Radiology, for interpretation of the barium meals and to Professor D. W. Piper for his encouragement and help in the preparation of this article.
References

Hayashida, T. (1969). End results of early gastric cancer collected from 22 institutions. Stomach and Intestine. Japan, 4, 1077-1085.

Kasumi, A. (1975). National Cancer Centre. Japan. (Personal communication.)

Kawai, K. (1971). Diagnosis of early gastric cancer. Endoscopy, vol. 3., No. 1.

Kidokoro, T. (1971). Frequency of resection, metastases and five-year survival rate of early gastric carcinoma in a surgical clinic. Gann Monograph on Cancer Research, 11, 45-49.

Murakami, T. (1971). Pathomorphological diagnosis. Gann Monograph on Cancer Research, 11, 53-55.

Muto, T. (1962). Factors influencing surficial results for gastric cancer. Nichushikaishi Japan, 47, 135-145.

Ransom, H. K. (1953). Cancer of stomach. Surgery, Gynecology, and Obstetrics, 96, 275-287.

Sano, R., and others (1972). Pathological evaluation of recurrence and mortality in early gastric cancer. Stomach and Intestine, 5, Japan, 531-540.

Segi, M. (1969). Cancer Mortality for Selected Sites in 24 Countries, no. 5 (1964-65), pp. 100-101. Department of Public Health: Tohoku University School of Medicine. Sendai, Japan.

Shirakabe, H. (1971). Double Contrast Studies of the Stomach. Bunkodo: Tokyo.

Sugano, H., Nakamura, K., and Takagi, K. (1971). An atypical epithelium on the stomach. A clinico-pathological entity. Gann Monograph on Cancer Research, 11, 257-269.

Yamada, K. T. (1975). A.I.C.H.I. Cancer Centre. Nagoya. (Personal communication.)

Yamada, E., Nakazato, H., Koike, A., Suzuki, K., Kato, K., and Kito, T. (1974). Surgical results for early gastric cancer. International Surgery, 59, 7-14.

Yokoyama, Y., Yokoyama, H., and Nagayo, T. (1974). On biopsy of excavated gastric lesions-with special reference to differential diagnosis of atypical regenerative and cancerous epithelia. Stomach and Intestine, vol. 9, no. 1, 9-20. 